\title{
ASSESMENT OF THE PROTON DONOR CAPACITY AS A MARKER OF ANTI-OXIDATIVE DEFENSE IN PREMATURE NEW BORNS
}

\author{
M. Matyas ${ }^{1}$, G. Zaharie ${ }^{1}$, M. Popa ${ }^{1}$, L. Blaga ${ }^{1}$, S. Andreica ${ }^{1}$, G. Vasile ${ }^{1}$, A. Muresan ${ }^{2}$ \\ ${ }^{1}$ Neonatology, ${ }^{2}$ Physiology, University of Medicine and Pharmacy, Cluj Napoca, Romania
}

Aim: The aim of this study was to determine the antioxidative defense in preterm by measuring the levels of proton donors (PD).

Material and methods: We conducted a prospective non-randomized study between March 2003 and December 2006. The study cohort was represented by twenty four premature newborns with diferent types of respiratory distress syndrome( RDS), asphyxia, cerebral hemorrhage. The control was represented by 13 healthy term newborns. For each newborn in the study group we determined the PD in the first and third days, while only on day one of life. Hatano's method was used to determine the PD. The statistical analysis was done using the „Statistica” program Ex- SPSS .

Results: In the study group median values of PD in the study group were 45.59\%[ 41.37-49.80] on day one and $49.01 \%[44.18-53.85]$ on day three with significant difference at corelation matrix $(\mathrm{p}<0.05)$.

The PD value on day one was significantly higher in premature newborns with medium RDS than in prematures with mild form of $\operatorname{RDS}(p=0.0065)$. The PD values on day one were significantly higher in the presence of cerebral hemorrhage $(\mathrm{p}=0.20)$.

On day one, the median PD in controls was significantly higher than that of the study group ( $\mathrm{p}=0,032699)$. There were no significant correlations between the values of the PD and the types of asphyxia.

Conclusions: The PD values increase on the first day life with RDS severity and presence of cerebral hemorrhage .At term newborns the PD value is significantly higher then in preterms with different patologies. 